\title{
Investigation of the Effect of Multimedia Education on Anxiety Before and After Surgery in Patients Undergoing Coronary Artery Bypass Graft Surgery
}

\section{Kosar Fahimi', Ali Abbasi², Mahdi Zahedi ${ }^{3}$ Farzaneh Amanpour ${ }^{4}$, Maryam Gilani', Hossein Ebrahimi ${ }^{6 *}$}

1. MSc Student in Critical Care Nursing, Student Research Committee, School of Nursing \& Midwifery, Shahroud University of Medical Sciences, Shahroud, Iran

2. Instructor, Department of Nursing, School of Nursing and Midwifery, Shahroud University of Medical Sciences, Shahroud, Iran

3. Assistant Professor, Ischemic Disorders Research Center, Golestan University of Medical Sciences, Gorgan, Iran

4. Instructor, Department of Statistics and Epidemiology, School of Public Health, Shahroud University of Medical Sciences, Shahroud, Iran

5. RN, Kordkuy Amiral-Momenin Hospital, Golestan University of Medical Sciences, Gorgan, Iran

6. Associate Professor, Randomized Controlled Trial Research Center, Shahroud University of Medical Sciences, Shahroud, Iran

\section{Article Info}

Received: $\quad 2018 / 01 / 7$

Accepted: $\quad$ 2018/02/7

Published Online 2018/03/16

DOI:

10.30699/sjhnmf.26.3.144

Original Article

Use your device to scan and read the article online

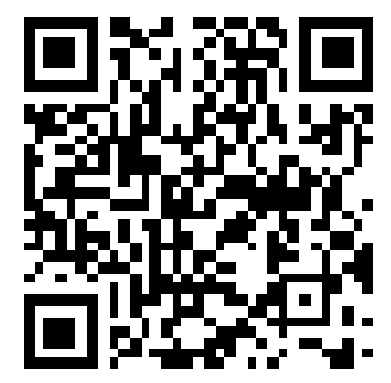

\section{Abstract}

Introduction: Anxiety is one of the prevalent events before and after surgery which occurs in patients due to the waiting time for heart surgery, hospitalization, fear of death, and in general the fear of unknowns. This study was conducted to investigate and compare the effect of multimedia education on anxiety before and after surgery in patients undergoing coronary artery bypass graft surgery.

Methods: In this randomized clinical trial where the data collector and the data analyzer were blinded, 110 patients undergoing coronary artery bypass graft surgery in 2016 were divided into two experimental and control groups (each group of 55) using quadruple blocking. For patients in experimental group, training through multimedia method was done and for the control group routine training was done. Patients' anxiety was evaluated using Spilberger's State-Trait Anxiety Inventory 5-7 days before surgery, and the day before surgery and the post-operative after extubation. Data was analyzed using descriptive and analytical statistics (Chi-square, and repeated measures analysis of variance).

Results: Both intervention and control groups were homogeneous in terms of demographic variables $(P<0.05)$. There was no significant difference between the mean of State-Trait Anxiety scores between two groups at three times $(P<0.05)$. The mean anxiety score of patients over time was significantly different, so the State, Trait, and total anxiety scores decreased significantly in the time of extubation compared to the previous two stages $(P<0.05)$.

Conclusion: Since, by using educational interventions the anxiety of patients was reduced compared to the preoperative situation, preoperative educational interventions are recommended to reduce the anxiety of these patients.

Keywords: Training by multimedia, Anxiety, Coronary Artery Bypass Graft

Copyright $($ C 2018, Sci J Hamadan Nurs Midwifery Fac. This is an open-access article distributed under the terms of the Creative Commons Attribution-noncommercial 4.0 International License which permits copy and redistribute the material just in noncommercial usages, provided the original work is properly cited.

How to Cite This Article:

Fahimi K, Abbasi A, Zahedi M, Amanpour F, Gilani M, Ebrahimi H. Inves-
tigation of the Effect of Multimedia Education on Anxiety Before and After
Surgery in Patients Undergoing Coronary Artery Bypass Graft Surgery. Sci J Hamadan Nurs Midwifery
Fac. 2018; 26 (3): 137-144




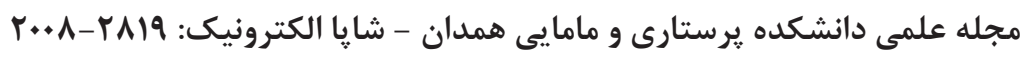

مقاله يزوهشى مامايى همدار

بررسى تأثير آموزش از طريق مولتىمديا بر اضطراب قبل و بعد از عمل بيماران تحت عمل جراحى عروق برإئى

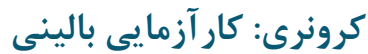

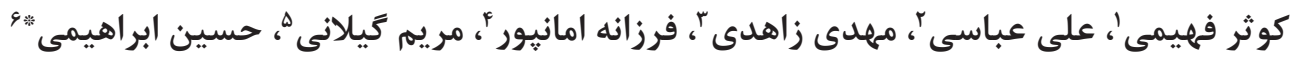

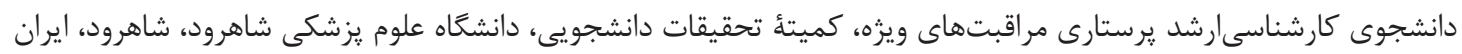

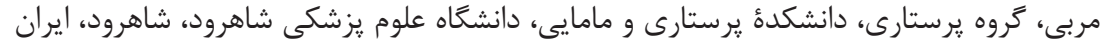

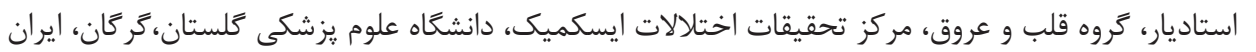

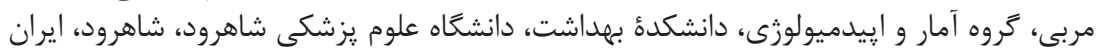

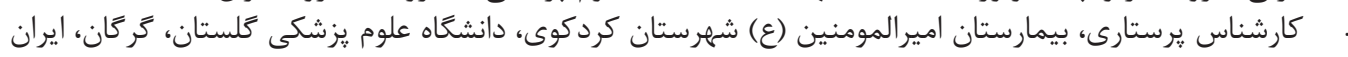

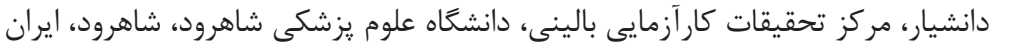

\begin{tabular}{|c|c|}
\hline קكيده & اطلاعات مقاله \\
\hline 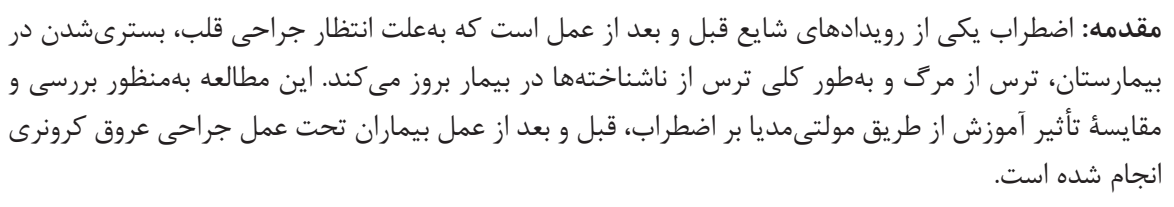 & 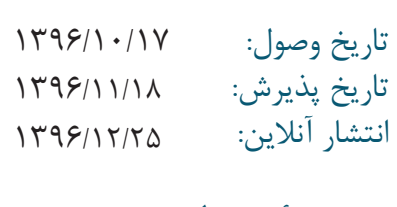 \\
\hline 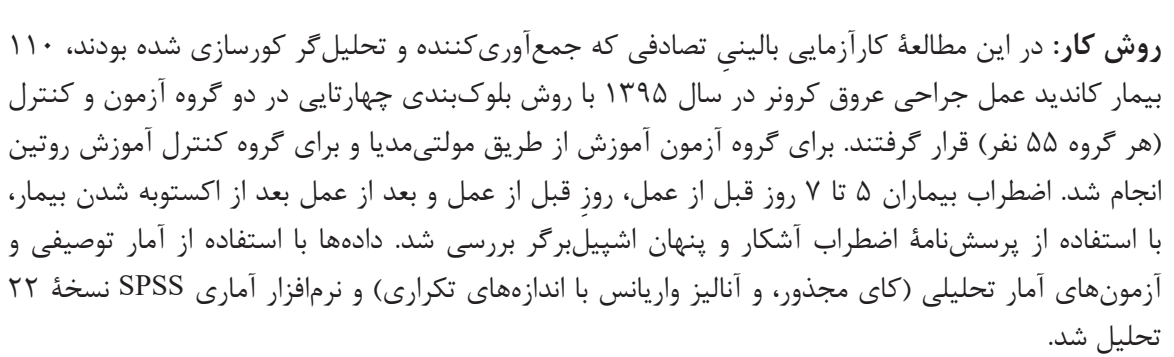 & 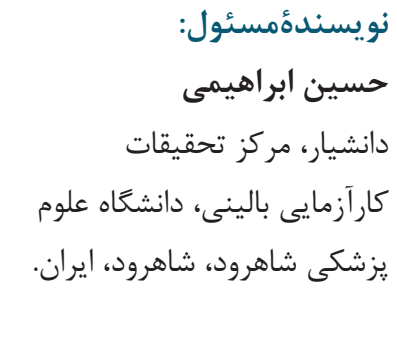 \\
\hline 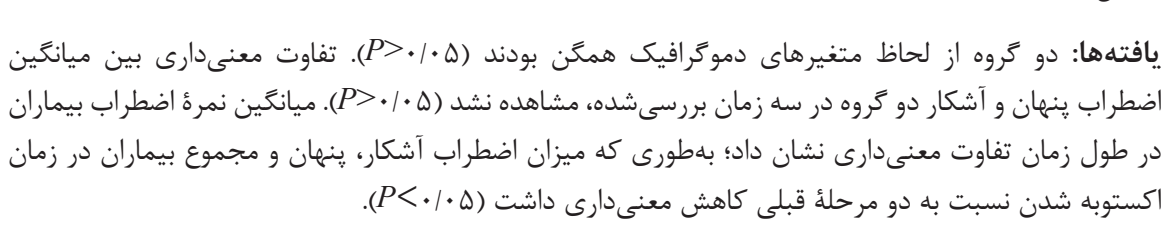 & يست الكترونيك: \\
\hline 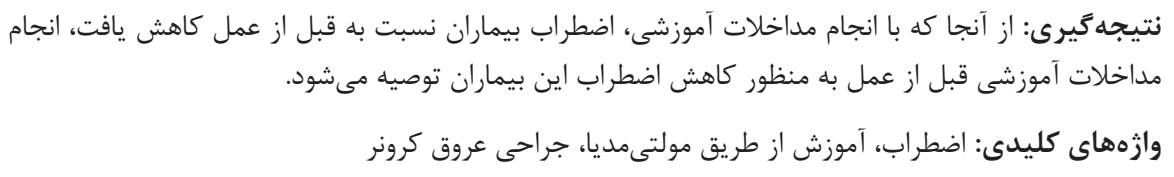 & \\
\hline
\end{tabular}

مقدمه

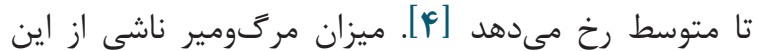

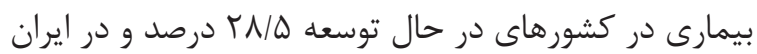

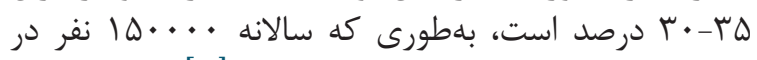

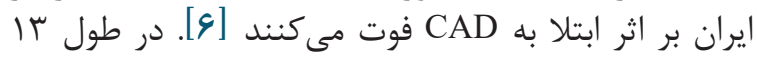

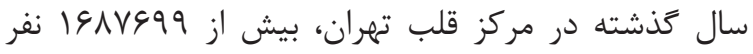

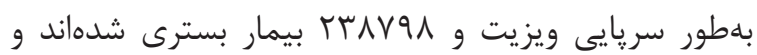

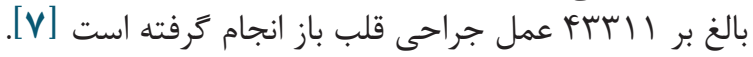

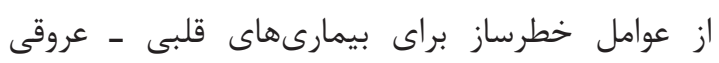

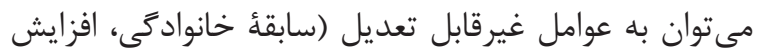

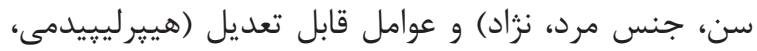

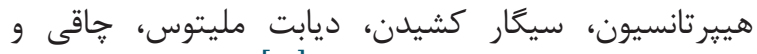

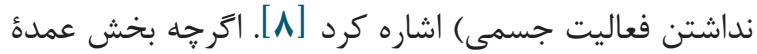

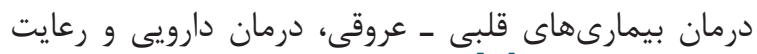
رزيم غذايى است [9]، جراحى هاى قلب دروفي و عروق، از جمله

دوره צץ شماره ץ - مرداد و شهريور Vqץו

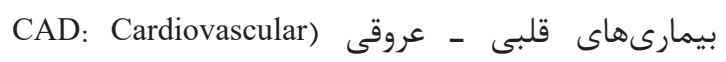
(Disease

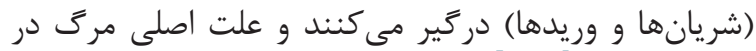

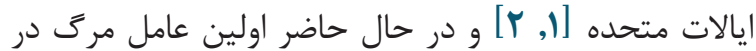

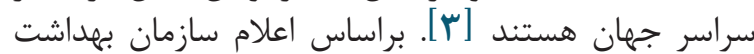

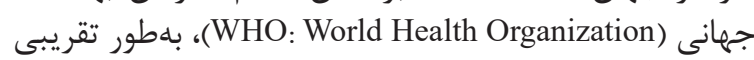

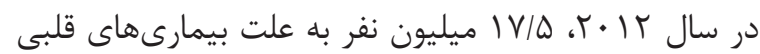

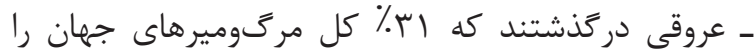

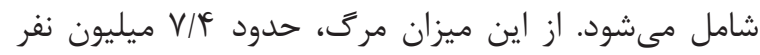

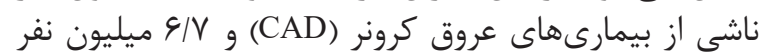

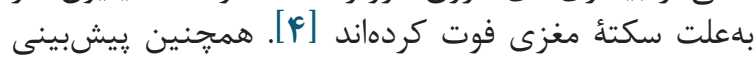

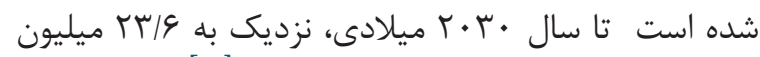

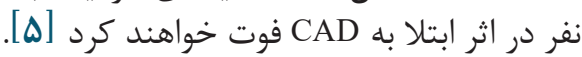

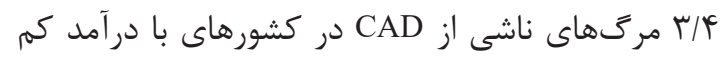
مجله علمى دانشكده يرستارى و مامايى همدان 


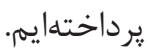 \\ روش كار}

اين يزروهش يك كار آزمايى بالينى تصادفى شده است و در

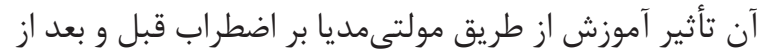

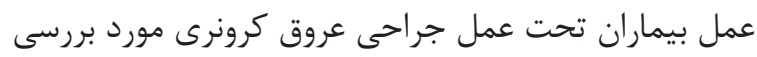

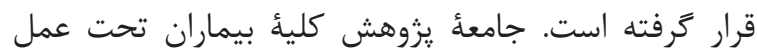

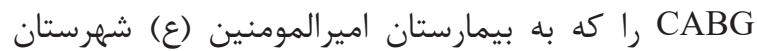

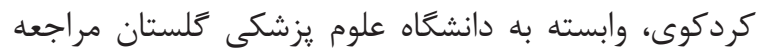

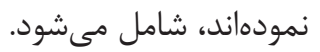

تعداد ها بيمار در هر كروه (آزمون و كنترل) از جامعأ

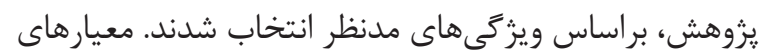

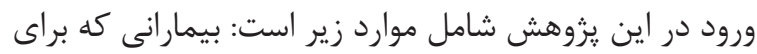

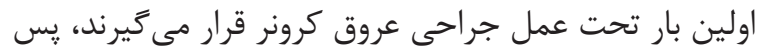

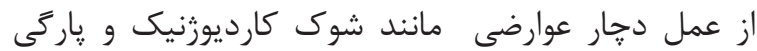

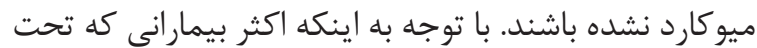

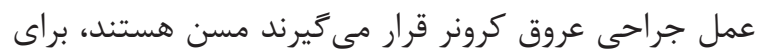

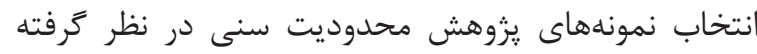

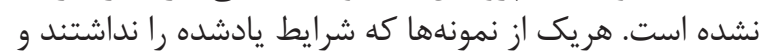

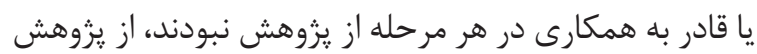

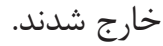

يس از انتخاب هريك از بيماران و توضيح هدف

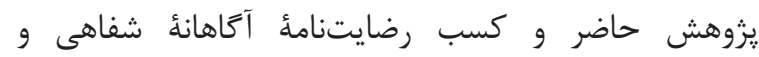
كتبى، شركت كنند

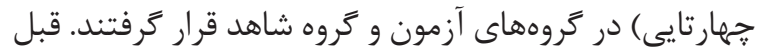

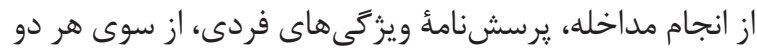

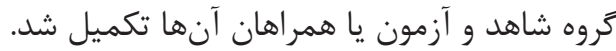

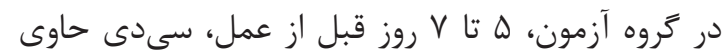

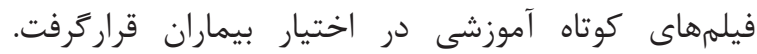

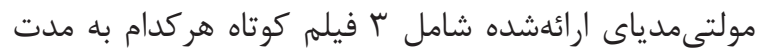

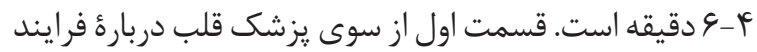

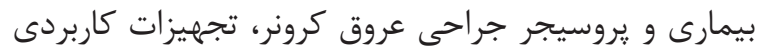

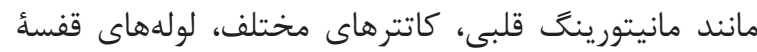

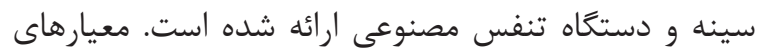

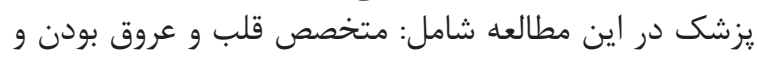

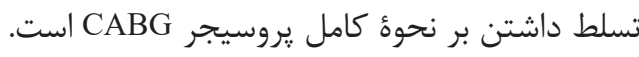

در قسمت دوم، يرستار اقدامات حمايتى يس إز از انجام

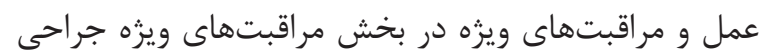

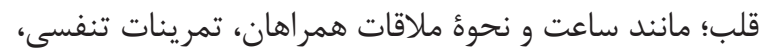

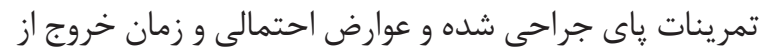

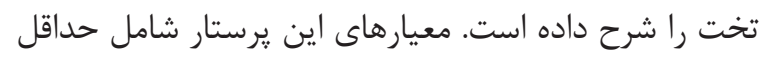

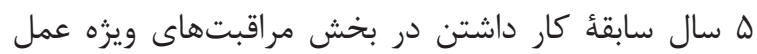
قلب باز و مسلط بودن بر تمرينات تنفسى و ساير موارد است.

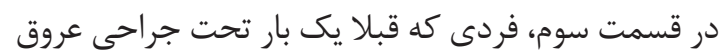

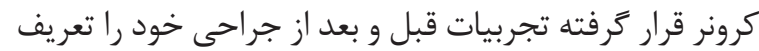

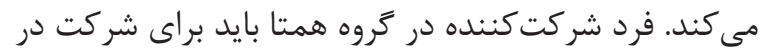

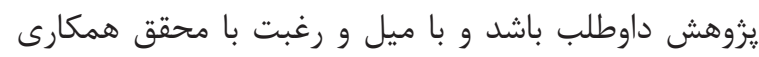

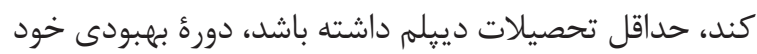

جراحى هاى متداولى هستند كه با هدف افزايش بقا و ارتقاى

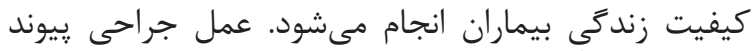

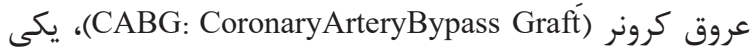
از راهكارهاى مهرم براى درمان بيمارى عروق كرونر استر

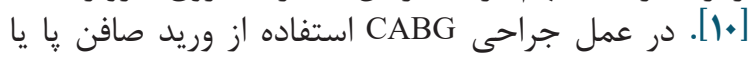

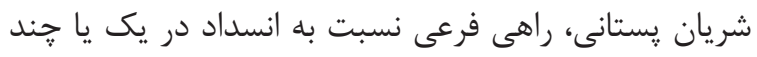

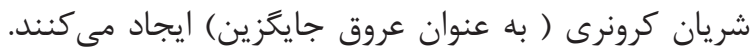

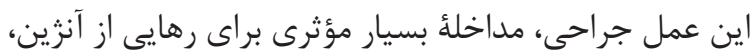

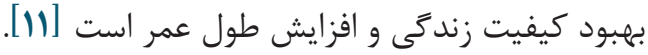

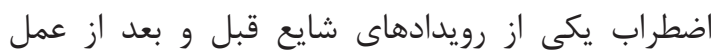

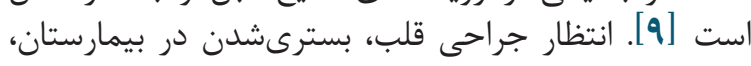

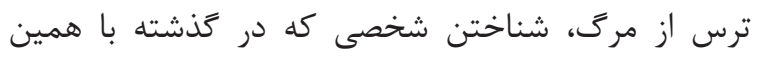

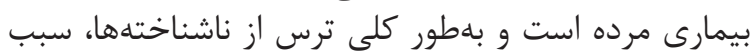

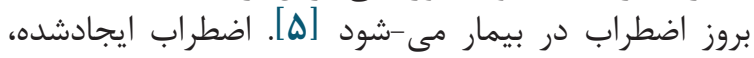

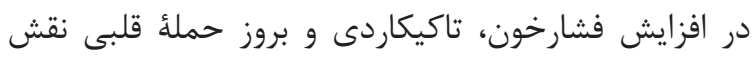

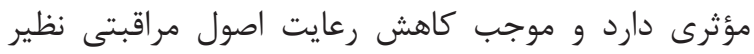

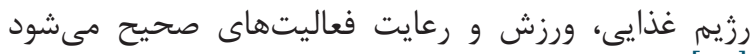

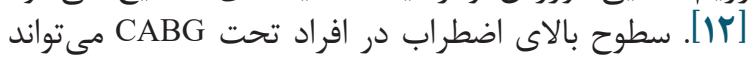

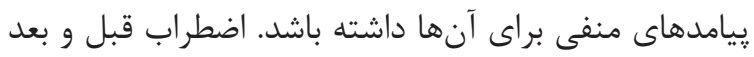

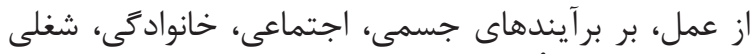

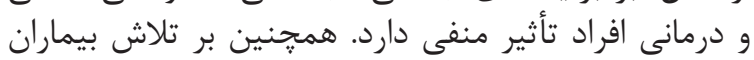

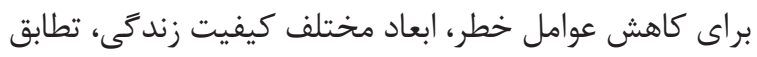

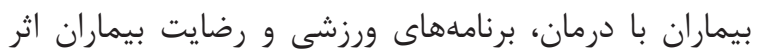

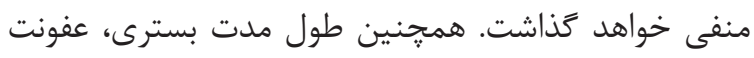

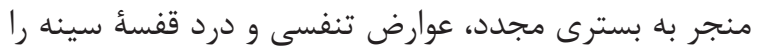

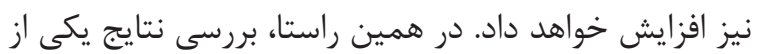

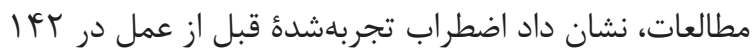

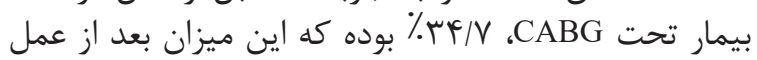

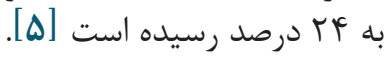

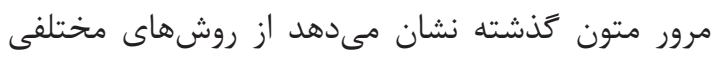

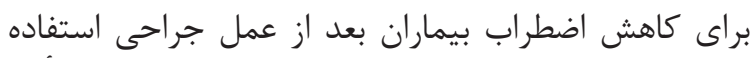

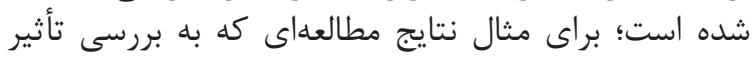

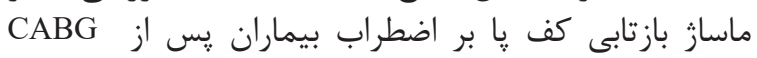

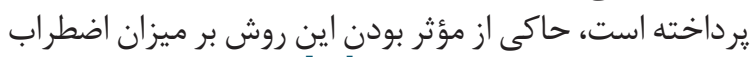

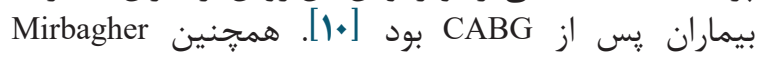

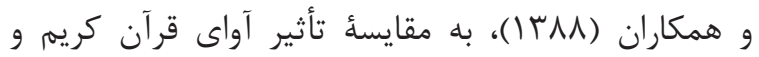

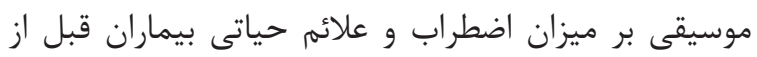

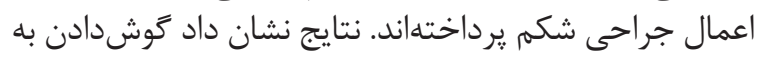

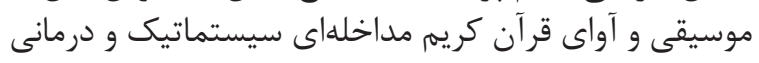

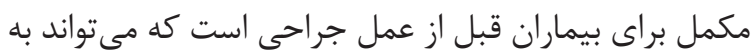

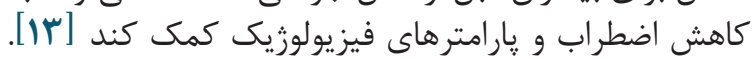

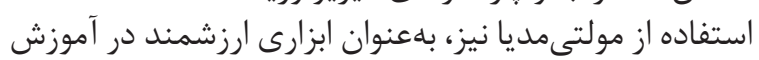

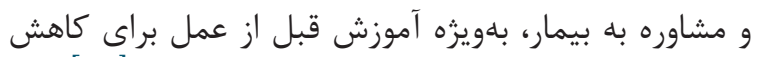

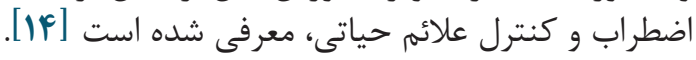

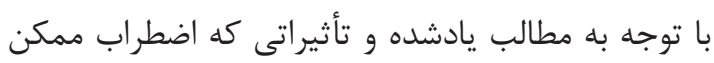

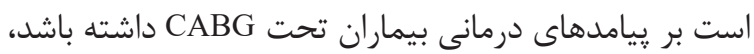

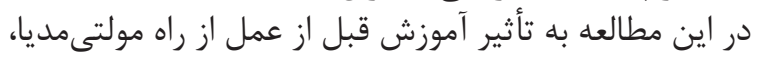

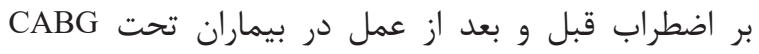




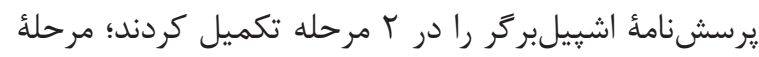

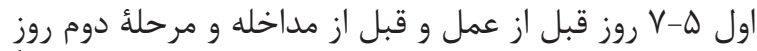

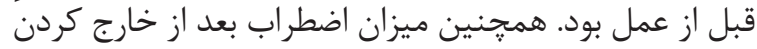

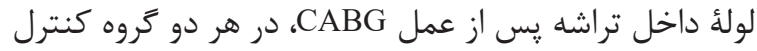

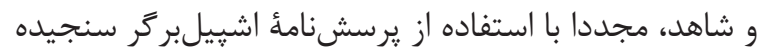

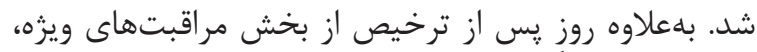

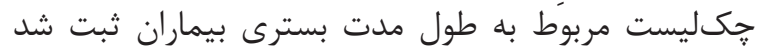

(تصوير ())

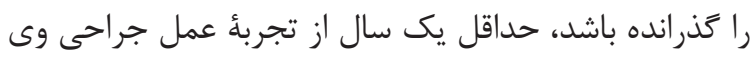

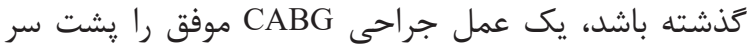

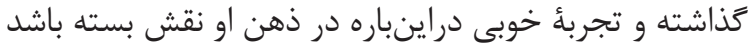

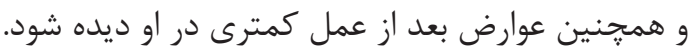
براى اطمينان از ديدن فيلم، بيماران بلهورت تلفنى

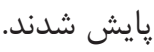
قبل از عمل، هر دو كروه براى تعيين ميزان اضطراب،

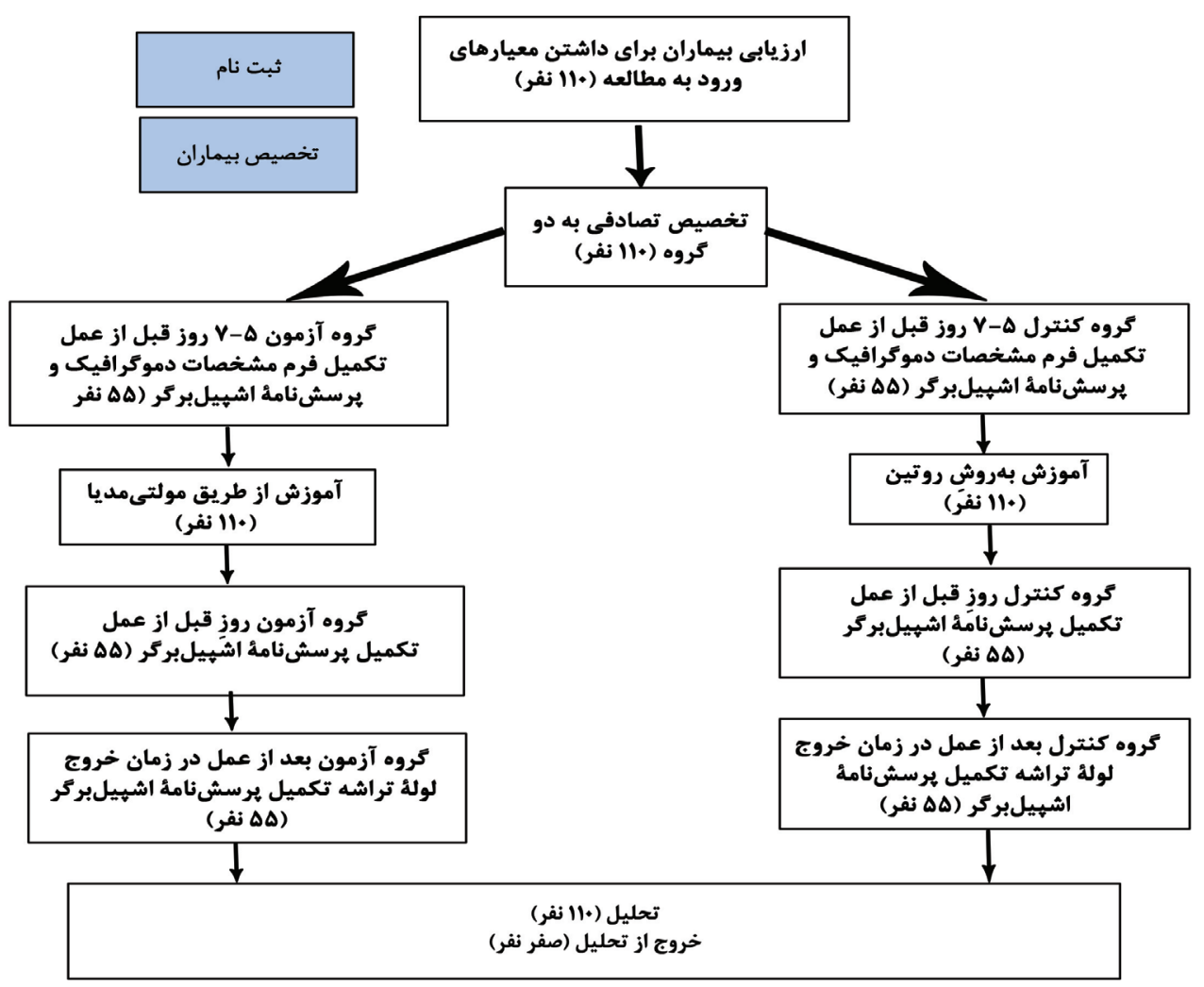

شكل ا. فلودياكر ام مطالعه

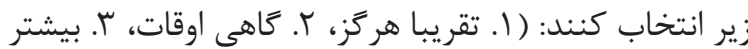

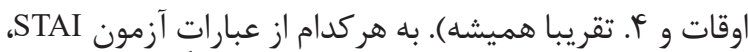

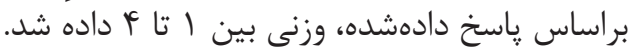

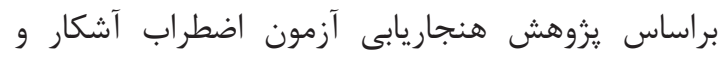

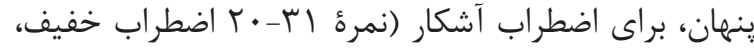

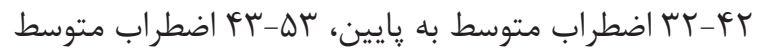

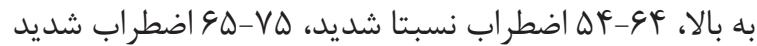

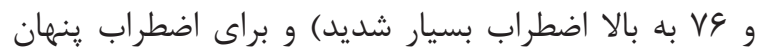

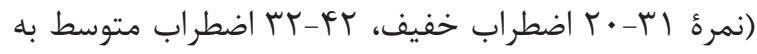
*ايين،

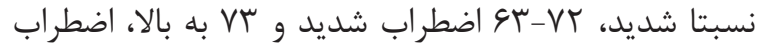

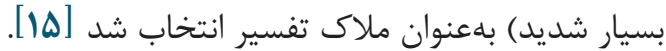

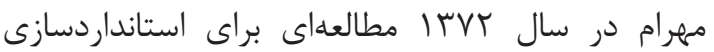

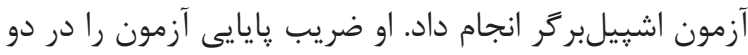

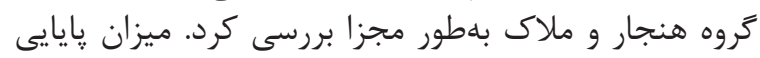

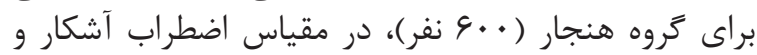

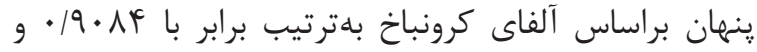

يرسشنامهُ ويزَّى هاى فردى شامل سؤالاتى دربارئ سن،

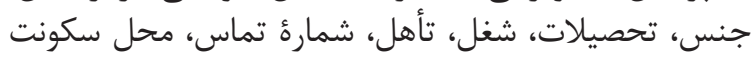
و وضعيت بيمه بوده است.

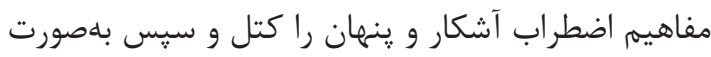

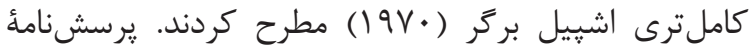

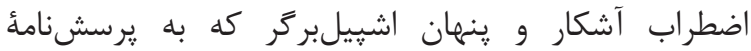

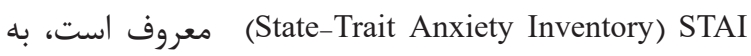
دفعات زياد در يزوهشها و فعاليتهاى بالينى استفاده شده

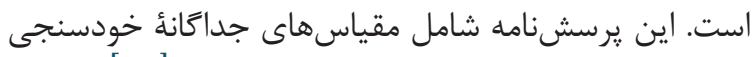

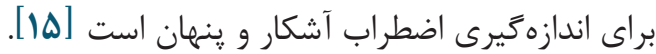

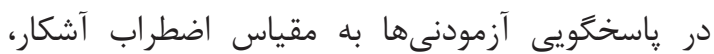

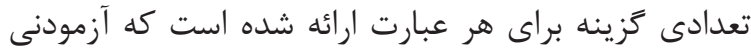

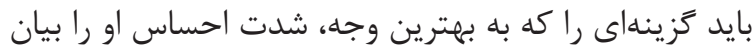

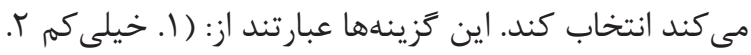

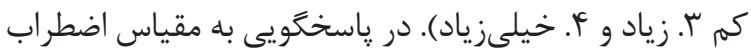

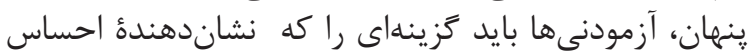

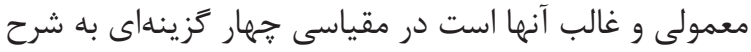




$$
\text { r ب در دو كروه كنترل و آزمون استفاده شد. }
$$

يافتهها

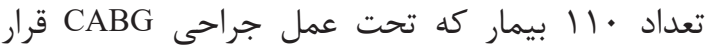

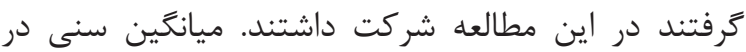

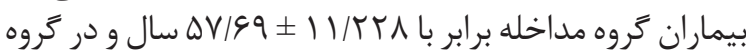

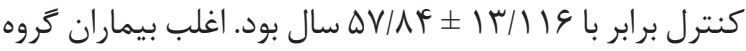

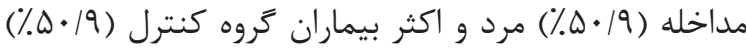

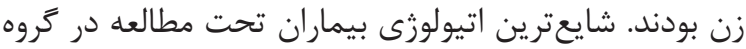

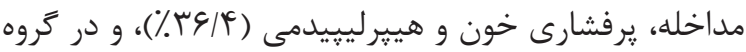

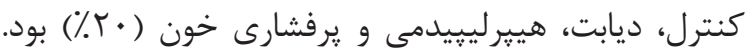

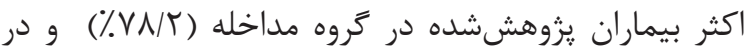

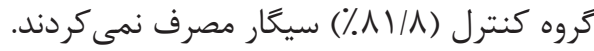

بر برسى مشخصات دموكرافيك نظير سن، جنس، وضعيت

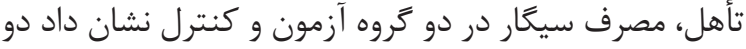

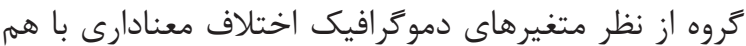

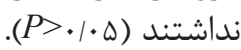

نتايج مقايسٔ ميانكَين اضطراب ينهان، آشكار و واضطراب

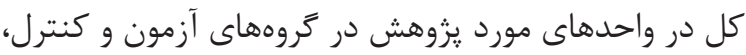

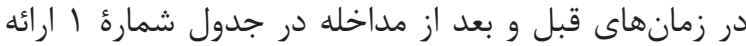

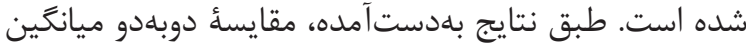

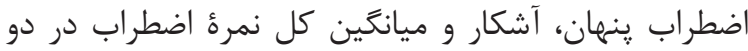

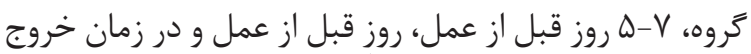

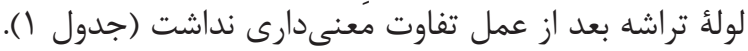

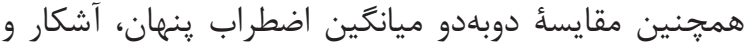

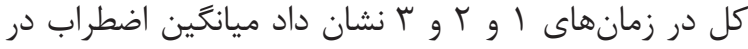

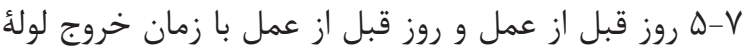

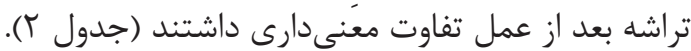

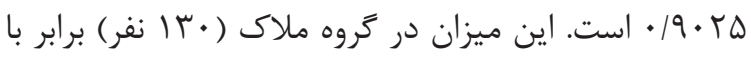

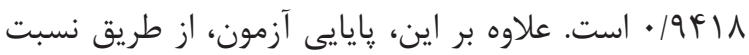

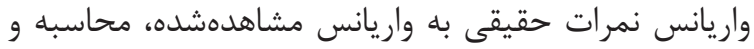

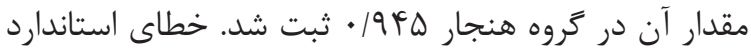

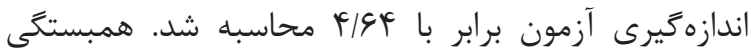

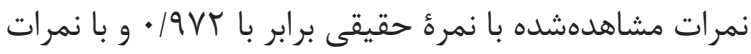

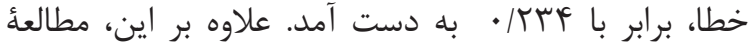

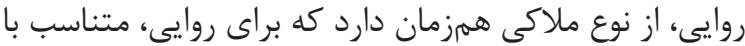

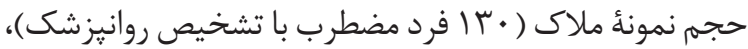

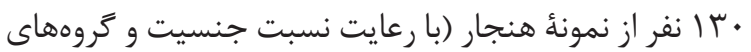

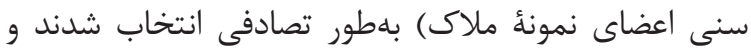

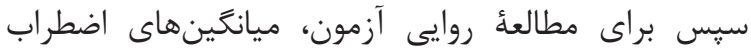

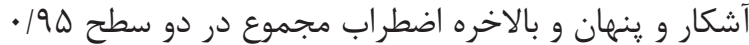

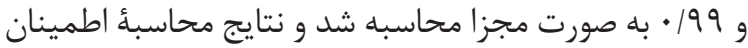

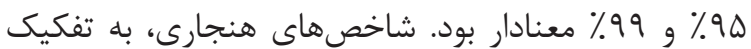

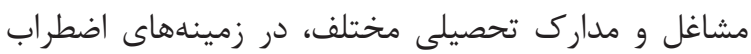

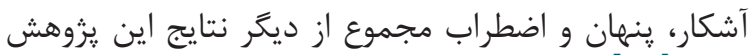

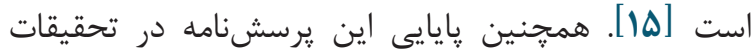

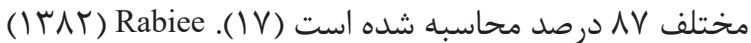

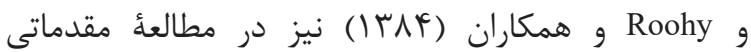

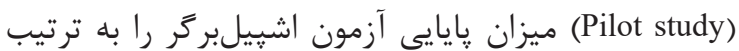

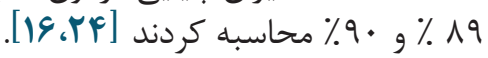

براى تعيين حجم نمونه، ميانكَين كَروه مورد، ميانكَين

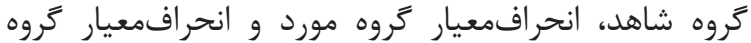

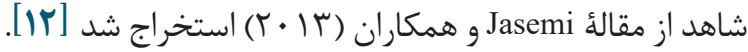
براى دستهبندى و خلاصه كردن يافتهها از آمار توصيفى

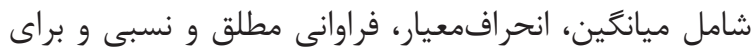

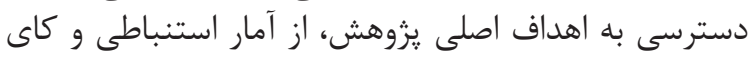

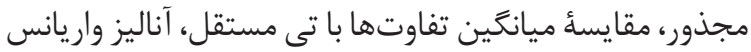
براى دادهاى تكرارى از طريق نرمافزار آمارى SPSS نسخة

\begin{tabular}{|c|c|c|c|c|}
\hline \multirow{2}{*}{ نتايج آزمون آناليز واريانس با اندازههاى تكرارى } & تروه شاهد & كروه آزمون & \multirow{2}{*}{ 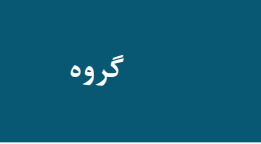 } & \multirow{2}{*}{ 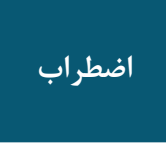 } \\
\hline & Mean \pm SD & Mean \pm SD & & \\
\hline \multirow{2}{*}{$\mathrm{F}=r / \cdot r q$} & FT/VTE q/Vr & $\kappa r / \backslash \Lambda \pm \Lambda / r V$ & هـ تا ح روز قبل از عمل & \multirow{3}{*}{ ضطراب آشكار } \\
\hline & $f \Delta / 9 \cdot \pm 1 \cdot 1 \cdot F$ & FT/Q९ $\pm N / T r$ & روزٍِ قبل از عمل & \\
\hline$P$ Value $=\cdot / / r \Delta$ & rI/৭9 $\pm V / r \Lambda$ & $r / / r q \pm 9 / 4 r$ & 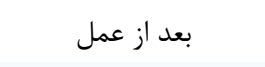 & \\
\hline \multirow{2}{*}{$\mathrm{F}=r / q V T$} & $r \cdot / r \pm N \cdot r$. & $r r / v \wedge \pm 1 \cdot / r r$ & هـ تا V روز قبل از عمل & \multirow{3}{*}{ ضطراب ينهان } \\
\hline & $K T / N F \pm N / T r$ & $r r / r \pm q / / r$ & روزٍ قبل از عمل & \\
\hline$P$ Value $=\cdot / \cdot \wedge 9$ & $r I / V F \pm 9 / 9$. & $r r / 9 \Delta \pm N / Y \varphi$ & بعد از عمل & \\
\hline \multirow{2}{*}{$\mathrm{F}=r / r T \Lambda$} & $\Delta r / q T \pm \mid r / \Lambda r$ & $\Lambda 9 / 99 \pm \mid V / 14$ & هـ تا ح روز قبل از عمل & \multirow{3}{*}{ اضطراب كل } \\
\hline & $\Lambda N / 9 \Delta \pm \mid G / T V$ & $19 / 19 \pm \mid F / F r$ & روزٍ قبل از عمل & \\
\hline$P$ Value $=\cdot / 11$. & $g r / V I \pm 11 / 9 \Delta$ & $q \mu / q 4 \pm \mid r / M \Lambda$ & 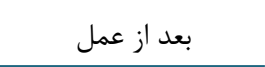 & \\
\hline
\end{tabular}

جدول شماره ا. توزيع ميانگين و انحر افمعيار ميزان اضطراب در زمانهاى قبل و بعد از مداخلهُ تروه آزمون و شاهد 
جدول شماره r. مقايسُٔ ميانغين و انحرافعيار اضطر اب در زمانهاى قبل و بعد از مداخله

\begin{tabular}{|c|c|c|c|c|c|}
\hline$P$ Value & خطاى معيار & تفاوت ميانگينها & $\mathbf{J}$ & I & نوع اضطراب \\
\hline . MTE & $\cdot / \Lambda$. &.$- / 91$ & بعد از مداخلئ قبل از عمل & ق ق قبل از مداخله & \multirow{3}{*}{ ينهان } \\
\hline$<\cdot / \cdot \cdot 1$ & $\cdot / \Lambda \Lambda$ & $\mathrm{V} / \mathrm{Vq}$ & بعد از مداخلئ بعد از عمل & قبل از مداخله & \\
\hline$<\cdot / \cdot \cdot 1$ & - IAT & $1 \cdot / \mathrm{Vr}$ & بعد از مداخلئ بعد از عمل & بعد از مداخله قبل از عمل & \\
\hline$\cdot \mid 9 \cdot \cdot$ & $\cdot / V \vee q$ & $-1 /$ FAT & بعد از مداخلئ قبل از عمل & قبل از مداخله & \multirow{3}{*}{ آشكار } \\
\hline$<\cdot / \cdot \cdot 1$ & $\cdot / \wedge \wedge \Delta$ & II/TYV & بعد از مداخلئ بعد از عمل & قبل از مداخله & \\
\hline$<\cdot 1 \cdot \cdot 1$ & $\cdot / A T \Delta$ & $\mid r / \Lambda \cdot 9$ & بعد از مداخلهُ بعد از عمل & بعد از مداخلهُ قبل از عمل & \\
\hline$\cdot / r \cdot$ & $1 / \pi r$. & $-Y / Y \& F$ & بعد از مداخلئ قبل از عمل & قبل از مداخله & \multirow{3}{*}{ كل } \\
\hline$<\cdot 1 \cdot \cdot 1$ & $|/ F| F$ & $-r \mid / 111$ & بعد از مداخلئ بعد از عمل & 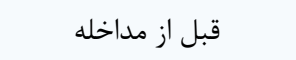 & \\
\hline$<\cdot 1 \cdot \cdot 1$ & $1 / 79 \Delta$ & $-r M / \Delta \wedge r$ & بعد از مداخلهُ بعد از عمل & بعد از مداخلهُ قبل از عمل & \\
\hline
\end{tabular}

اختلاف آمارى معنادارى وجود نداشته و دو گروه از اين نظر

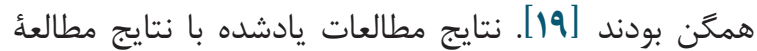

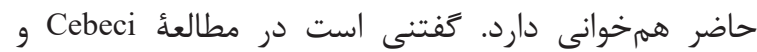

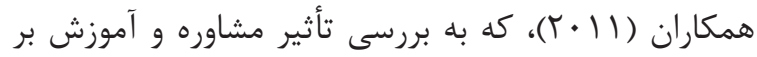

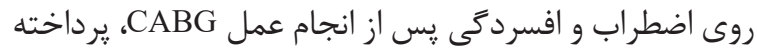

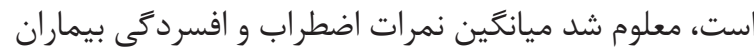

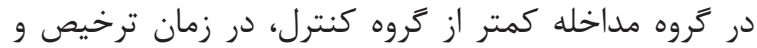

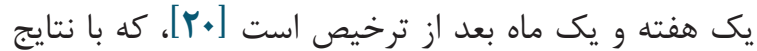

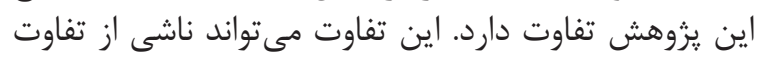

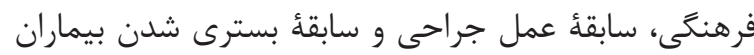

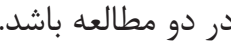

در اين مطالعه اضطراب ينهان، آشكار و كل، در بيماران

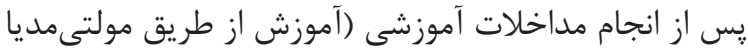

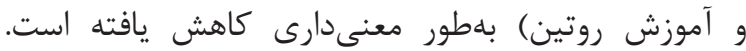

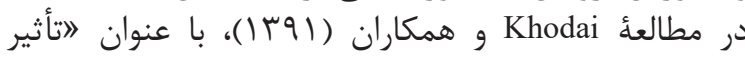

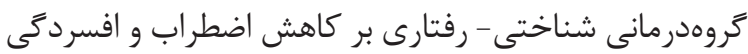

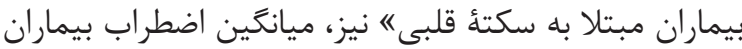

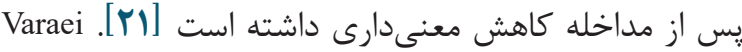

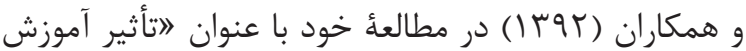

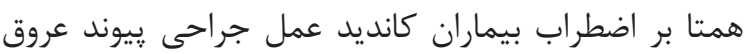

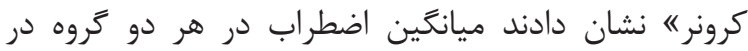

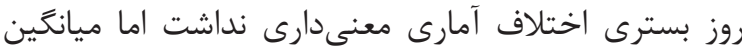

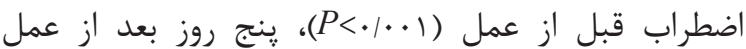
ماخله

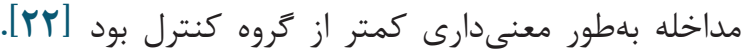

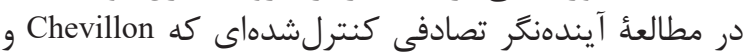

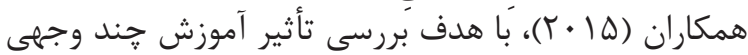

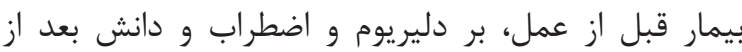

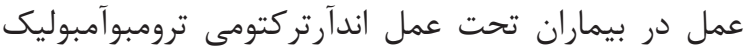

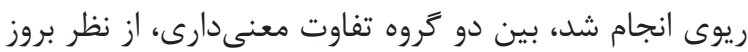

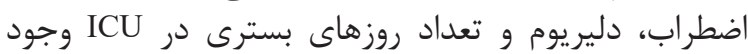

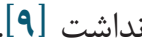

نتايج يزوهش Delaney و همكاران (•l· ( ) كه با هدف

\section{بحث}

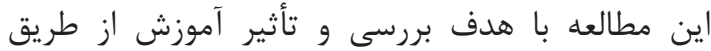

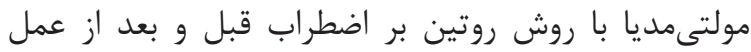

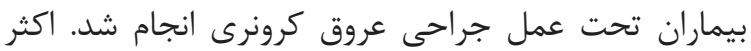

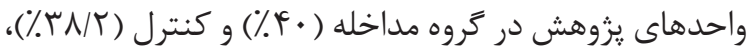

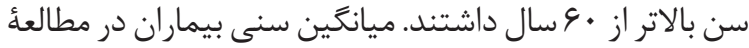
Chevillon

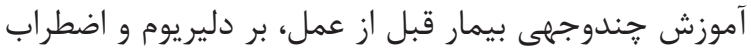

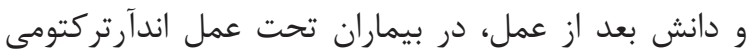

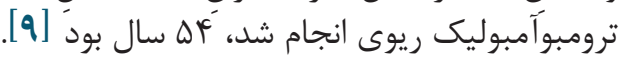

در اين مطالعه، بيماران از نظر جنسيت همخَن بوان بودند.

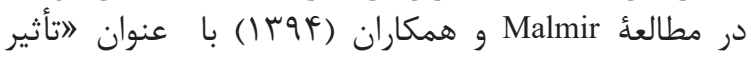
آرامسازى بنسون بر سطح اضطراب آشكار بيماران كانديد

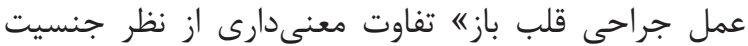

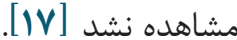

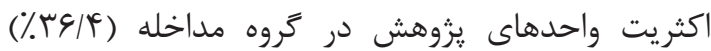

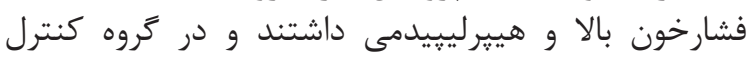

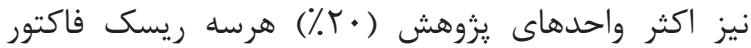

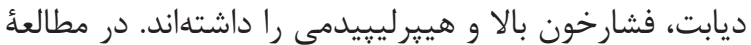
Shini Jabery

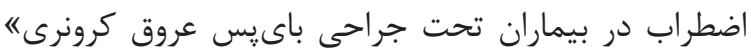

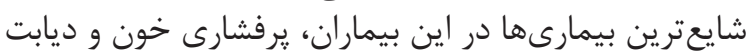
مليتوس ذكر شده است [ه إن ].

در اين مطالعه، در بررسى ميانكين اضطراب رينهان، آشكار

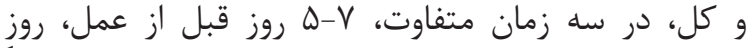

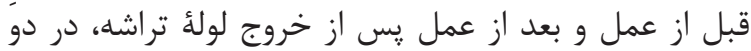

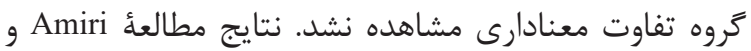

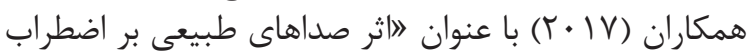

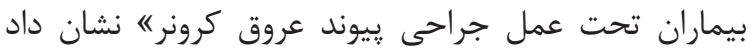

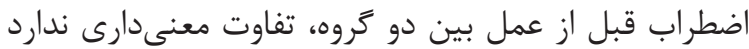

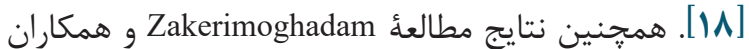

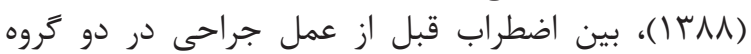


انجام مداخله براى گَروه آزمون در محلى كه بيماران گروه

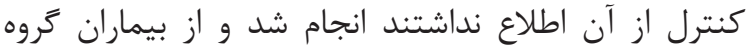

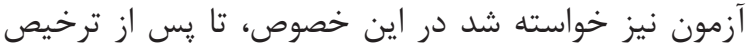

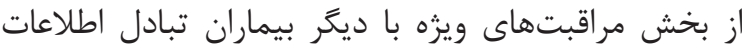
نكند . بحت

\section{سياسگزارى}

اين مطالعه بخشى از نتايج زايان نامهُ دانشجويى مقطع

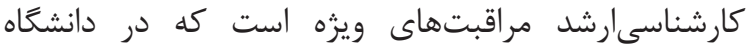

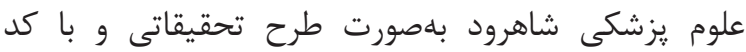

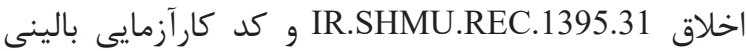
IRCT2016062828686N1 يثزوهشگران از معاونت يزوهشى، سريرستار و كاركنان محترم

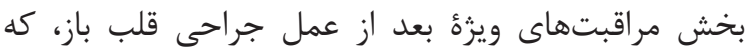

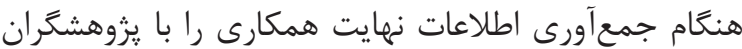

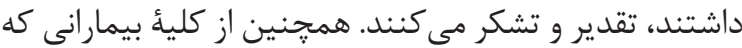

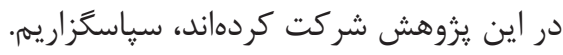

$$
\text { تعارض منافع }
$$

بين نويسند
بررسى تأثير مداخلأ مبتنى بر معنويت بر كيفيت زندكى،

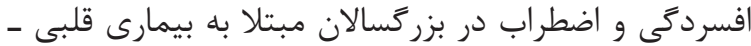

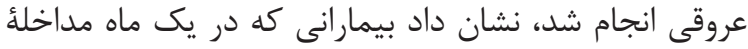

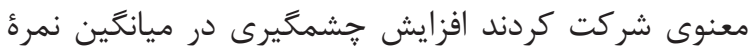

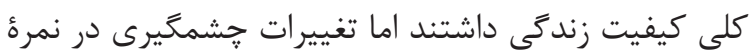

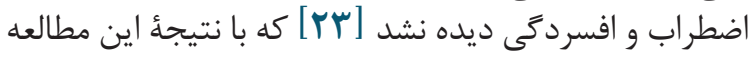

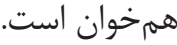

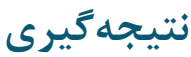

براساس نتايج مطالعه، آموزش از طريق مولتى مديا نسبت

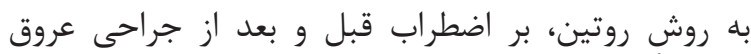

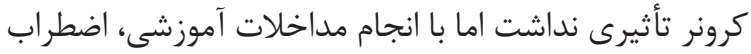

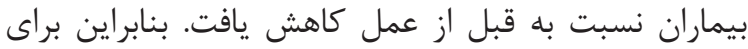

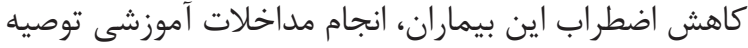

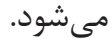

\section{محدوديتهاى يزوهش}

احتمال كسب اطلاعات از سوى بيماران از ديخر منابع،

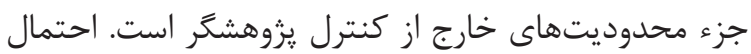

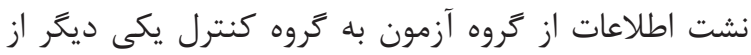
محدوديتهاى اين مطالعه بود. براى تقليل اين محدوديت،

\section{References}

1. Mattson CMC. Depression, Anxiety, and Social Support Fail to Predict Heart Rate Recovery in Exercise Stress Test Patients: Kent State University; 2011.https://etd.ohiolink.edu/rws_etd/document/get/kent1302305453/inline

2. Roger VL, Go AS, Lloyd-Jones DM, Benjamin EJ, Berry JD, Borden WB, et al. Heart disease and stroke statistics - 2012 update. Circulation. 2012;125(1):e2-e220.https://doi.org/10.1161/ $\underline{\text { CIR.0b013e31823ac046 }}$

3. Loscalzo J. Harrison's Cardiovascular Medicine: McGraw-Hill Education; 2010.

4. World Health Organization. Cardiovascular diseases (CVDs) 2016.

5. Shini Jabery P, Biranvand S, Baraz S, Asadi S. Level of anxiety in patients undergoing coronary artery bypass graft surgery. Aflak. 2011;7(2425):8.

6. Babaee G, Keshavarz M, Shayegan AH. Effect of a health education program on quality of life in patients undergoing coronary artery bypass surgery. Acta Medica Iranica. 2007;45(1):69-75.

7. Abbasi SH, Kassaian SE, Sadeghian S, Karimi A, Saadat S, Peyvandi F, et al. Introducing the Tehran Heart Center's Premature Coronary Atherosclerosis Cohort: THC-PAC Study. The Journal of Tehran University Heart Center. 2015;10(1):34-42.
8. Asaee R, Nasari H, Hoseini S. Prevalence of delirium in hospitalized internal medicine and surgical adult patients in Shohadaye ashayer hospital of Khoram abad. scientific magazine yafte. 2008;10(3):21-7.

9. Chevillon C, Hellyar M, Madani C, Kerr K, Kim SC. Preoperative education on postoperative delirium, anxiety, and knowledge in pulmonary thromboendarterectomy patients. Am J Crit Care. 2015;24(2):164-71.https://doi.org/10.4037/ ajec2015658

10. Raygani Visi AA, Ahmadi M, Rezae M, B H, Taghizadeh P. The Effect of Foot Reflexology on Anxiety after Coronary Artery Bypass Graft. Arak Medical University Journal. 2014;16(12):87-95.

11. Asgary M, Soleimani M. Comprehansive book intensive nursing care in ICU,CCU and dialysis wards. 1, editor. Tehran: Boshra; 2011.

12. Jasemi M, Rahmani A, Aghakhani N, Eghtedar S, Alinejad $\mathrm{H}$. The effect of face to face education on anxiety and hemodynamic status of patients who are candidates for CABG surgery. Razi Journal of Medical Sciences. 2013;20(114):28-34.

13. Mirbagher Ajorpaz N, Aghajani M. The effects of music and Holy Quran on patients' anxiety and vital signs before abdominal surgery. Evidence Based Care. 2011;1(1):63-76.

14. Ihrig A, Herzog W, Huber CG, Hadaschik B, Pahernik S, Hohenfellner M, et al. Multimedia sup- 


$$
\text { FF F أ. بررسى تأثير آموزش از طريق مولتىمديا بر اضطراب... }
$$

port in preoperative patient education for radical prostatectomy: the physicians' point of view. Patient Educ Couns. 2012;87(2):239-42.https://doi. org/10.1016/j.pec.2011.08.014

15. Behdani F, Sargolzaei MR, Ghorbani E. Study of the relationship between lifestyle and prevalence of depression and anxiety in the students of Sabzevar Universities. 2000.

16. Roohy GR, Rahmany A, Abdollahy AA, Mahmoody GhR. The effect of music on anxiety level of patients and some of physiological responses before abdominal surgery. Journal of Gorgan University of Medical Sciences. 2005;7(1):75-8.

17. Malmir M, Teimouri F, Pishgooie A, Dabaghi P. The Role of Benson's relaxation on reducing state anxiety on candidate of open heart surgery patient's . Military Caring Sciences. 2015;2(3):18290.https://doi.org/10.18869/acadpub.mcs.2.3.182

18. Amiri MJ, Sadeghi T, Bonabi TN. The effect of natural sounds on the anxiety of patients undergoing coronary artery bypass graft surgery. Perioperative Medicine. 2017;6(1):17.https://doi. org/10.1186/s13741-017-0074-3

19. Zakerimoghadam M, Aliasgharpoor M, Mehran A, Mohammadi S. Effect of Patient Education about Pain Control on Patients' Anxiety Prior to Abdominal Surgery. Hayat. 2010;15(4):13-22.
20. Cebeci F, Çelik SS. Effects of discharge teaching and counselling on anxiety and depression level of CABG patients. 2011.

21. Khodai S, Khazai K, Ali Abadi Z, Kazemi T. The Effect of Cognitive-Behavioral Group Therapy on Depression and anxiety in Patients with myocardial infarction. Modern Care Journal (Scientific Quarterly of Birjand Nursing \& Midwifery Faculty). 2012;9(4):364-70.

22. Varaei Sh, Cheraghi MA, Seyedfatemi N, Talebi M, Bahrani N, Dehghani A, et al. Effect of peer education on anxiety in patients candidated for coronary artery bypass graft surgery: a randomized control trial. Journal of Nursing Education. 2013;2(3):28-37.

23. Delaney C, Barrere C, Helming M. The influence of a spirituality-based intervention on quality of life, depression, and anxiety in community-dwelling adults with cardiovascular disease: a pilot study. J Holist Nurs. 2011;29(1):21-32. https://doi.org/10.1177/0898010110378356

24. Rabiee M, Kazemi Malek Mahmodi S, Kazemi Malek Mahmodi S. The effect of music on the rate of anxiety among hospitalized children. Journal of Gorgan University of Medical Sciences. 2007;9(3):59-64. 